\title{
COPD and bronchodilators: should the heart pay the bill for the lung?
}

\author{
Nera Agabiti (i) ${ }^{1}$ and Giuseppe Maria Corbo ${ }^{2}$ \\ Affiliations: ${ }^{1}$ Dept of Epidemiology, Lazio Regional Health Service, Rome, Italy. ${ }^{2}$ Pulmonary Medicine Dept, \\ Catholic University, Rome, Italy.
}

Correspondence: Nera Agabiti, Dept of Epidemiology, Lazio Regional Health Service, Via Cristoforo Colombo 112, Rome 00147, Italy. E-mail: n.agabitidadeplazio.it

@ERSpublications

Treatment with bronchodilators in COPD patients should take into account cardiovascular comorbidities http://ow.ly/gMIG30aAwTK

Cite this article as: Agabiti N, Corbo GM. COPD and bronchodilators: should the heart pay the bill for the lung? Eur Respir J 2017; 49: 1700370 [https://doi.org/10.1183/13993003.00370-2017].

\begin{abstract}
The question
The pharmacological treatment of patients with coexisting cardiovascular disease (CVD) and chronic obstructive pulmonary disease (COPD) is challenging, because some drugs for COPD patients should be used with caution in patients with CVD, and vice versa. The crucial question on cardiovascular safety of long-acting bronchodilators, i.e. long-acting $\beta_{2}$-agonists (LABAs) and long-acting anti-muscarinic agents (LAMAs, principally tiotropium), has no definitive answer. Because of different mechanisms of action, the combination of these drugs is expected to be more effective than the individual components in the maintenance treatment of COPD [1]. Evidence available from randomised controlled trails and observational studies on increased risk of cardiovascular adverse events is large but difficult to summarise because of complex methodology, different comparison groups and potential bias in each study [2-5]. Results are controversial and inconclusive. The risks of combining LABAs and tiotropium for the treatment of COPD are still unclear. Whether COPD patients treated with long-acting bronchodilators have increased risk of heart failure, or patients with both COPD and heart failure have higher risk of adverse events, remain open questions.
\end{abstract}

\section{What the study adds}

In this issue of the European Respiratory Journal, the study presented by SuISSA et al. [6] interestingly contributes to the ongoing debate. The focus is on the concurrent use of long-acting bronchodilators. A large COPD patient cohort was selected from the UK Clinical Practice Research Datalink for 2002-2012 and followed up over 1 year. Compared with monotherapy, adding a second long-acting bronchodilator (either a LABA or tiotropium) did not increase the risk of myocardial infarction, stroke or arrhythmia. However, a statistically significant $16 \%$ increase in the risk of heart failure was found with the addition of a second long-acting bronchodilator rather than monotherapy; the increase was higher (23\%) when patients with previous diagnosis of heart failure (about $3 \%$ of the whole study population) were excluded. Large numbers from real-world settings, sophisticated analytical approaches and sensitivity analyses led to robust results, considering the known limits of observational studies on the comparative effectiveness and safety of drugs. The fact that the use of two long-acting bronchodilators was found to be safe, in terms of major cardiovascular events, is reassuring and supports the guidelines/recommendations regarding the use of fixed-dose combination bronchodilators. However, the effect on heart failure is relevant and deserves some additional consideration.

Received: Feb 212017 | Accepted: Feb 222017

Conflict of interest: None declared.

Copyright OERS 2017 


\section{Relationship between COPD and CVD}

Heart-lung interactions are complex, particularly in the context of heart failure. CVD is undoubtedly the most significant non-respiratory contributor to both morbidity and mortality in COPD. The prevalence of CVD in patients with COPD was found to range from $7.1 \%$ to $31.3 \%$ for heart failure, from $4.7 \%$ to $60 \%$ for ischaemic heart disease, from $0.3 \%$ to $29 \%$ for arrhythmia and from $6.9 \%$ to $9.9 \%$ for stroke $[7,8]$. The strong overlap between the two conditions is due to shared similar risk factors, such as ageing, smoking, exposure to environmental pollution, metabolic disorders and altered inflammatory response [7, 9]. On the one hand, the increased risk of CVD persists after adjustment for common risk factors, suggesting that COPD plays a role in the development of CVD [7]. On the other hand, COPD patients with a more severe reduction of forced expiratory volume in $1 \mathrm{~s}$ have a greater risk of occurrence of heart failure, atrial fibrillation and myocardial infarction [7]. COPD is frequent and often undiagnosed among patients with heart failure (with rates from $13 \%$ to $38 \%$ ) [10] and is associated with higher mortality [11]. Over the last few years, researchers and clinicians have focused on the autonomic nervous system, which is impaired in both COPD and CVD [12]. Heart rate variability is frequently used as a measure of autonomic control. It has been found that COPD patients have a reduced heart rate variability [13] and that autonomic control during exercise is altered [14]. A reduction in heart rate variability reflects a sympathetic activation; this is a prognostic factor in ischaemic heart disease [15], heart failure, hypertension and stroke [16]. Clinical trials on the safety of these drugs on cardiovascular autonomic control have been performed $[17,18]$, but the absence of adverse effects could not be excluded, because of the selection of samples and the short duration of the studies. The complex relationship between CVD and COPD is summarised in figure 1.

\section{Relationship between COPD and heart failure}

Heart failure is a complex syndrome owing to the inability of the heart to pump sufficient blood to meet the needs of the body's tissues. It represents the end stage of cardiac disease, mostly following coronary disease or hypertension. Two distinct clinical syndromes have been identified: heart failure with reduced ejection fraction ( $\mathrm{HFrEF}$ ), i.e. $\mathrm{EF}<40 \%$, which is characterised by abnormalities in left ventricular systolic function, progressive chamber dilation and eccentric remodelling, and heart failure with preserved ejection fraction (HFpEF), i.e. $\mathrm{EF} \geqslant 50 \%$, which presents normal or near normal systolic function and evidence of diastolic dysfunction with concentric remodelling or hypertrophy [19]. Heart failure with mid-range EF (i.e. EF range $40 \%-49 \%$ ) has recently been labelled [19]. About half of patients with heart failure have a normal ejection fraction [20].

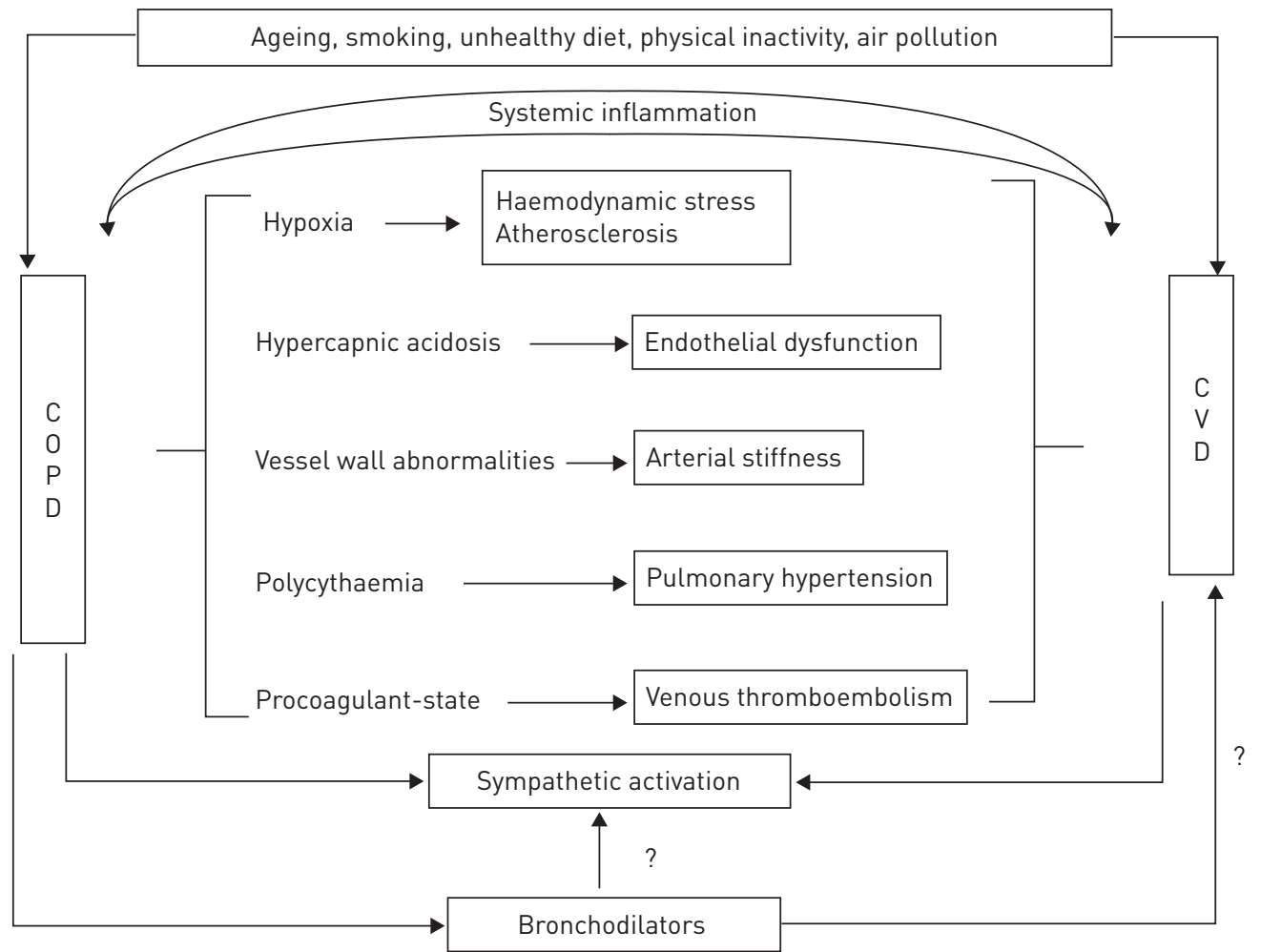

FIGURE 1. The complex interaction between chronic obstructive pulmonary disease (COPD) and cardiovascular disease (CVD). 
Although patients with heart failure present similar clinical signs and symptoms, it is important to distinguish the various forms of heart failure because they differ in pathophysiology, structural changes in the cardiac muscle, neuro-humoral and inflammatory molecular mechanisms, epidemiology, potential susceptibility to environmental triggers and response to treatment [19]. Some biomarkers have been linked to the heart failure subtypes. For example, plasma B-natriuretic peptide concentration in patients with HFpEF was lower than in patients with HFrEF, and highly sensitive troponin $\mathrm{T}$ was significantly associated with development of HFrEF; in contrast, growth factor GDF, cystatin C and urinary albumin excretion were significantly associated with the development of HFpEF [21]. Opportunities and challenges of old and new pharmacological treatments of heart failure patients have been updated in the clinical guidelines [22]. However, the role of both beta-blockers and ACE/ARB (angiotensin-converting enzyme/angiotensin receptor blocker) in HFpEF is not well established and there is no evidence-based optimal therapeutic protocol for HFpEF [22]

In this respect, the finding from the study by SuIssA et al. [6], that concomitant use of long-acting bronchodilators influences the incidence of new heart failure (hazard ratio 1.21 among those with no heart failure diagnosis before cohort entry), suggests that these drugs play a role in the development of heart failure in COPD patients in a 1-year follow-up period. Accurate identification of COPD subgroups that are potentially more vulnerable to cardiac drug toxicity is crucial. Measuring coexisting cardiac diseases, subtypes and severity of heart failure, conditions/comorbidities associated with poor prognosis such as peripheral artery disease or diabetes, and severity of lung function impairment is challenging, but cannot be fully achieved in large observational studies based on healthcare electronic databases. Because clinical presentation is fundamental to the diagnosis of heart failure, and heart failure and COPD share both risk factors and clinical presentation, making the correct diagnosis may be difficult [23]. Acute respiratory symptoms may have mixed pulmonary and cardiac origin [24]. Echocardiography is the cornerstone for the diagnosis of heart failure, but in patients with pulmonary emphysema, air trapping may alter echocardiographic acoustic windows, leading to poor image quality in many COPD patients [25]. Moreover, N-terminal pro-brain natriuretic peptide may improve the diagnostic accuracy of heart failure in stable COPD [26].

Rigorous measurement of drug use that is potentially dangerous for the heart is another crucial point in pharmacological safety research, with a particular focus on potential interaction between drugs and a consideration of patients' adherence to medical prescription and how this changes over time. The increased risk of heart failure resulting from the addition of a LABA rather than tiotropium (hazard ratio 1.28 versus 1.11) compared with the risk of monotherapy is another interesting finding from the work of Suissa et al. [6], and the hypothesis of a trigger action of LABAs in precipitating heart failure cannot be ruled out. Longer follow-up studies could elucidate this aspect. Mechanisms for decompensated heart failure are complex and not fully understood. Multiple risk factors have been proposed and include ischaemia, arrhythmia, respiratory infections, COPD exacerbations and air pollution [19, 27].

\section{Conclusions}

Suissa et al. [6] have added important data to the ongoing debate on the cardiac safety of long-acting bronchodilators. Given the rapid development of new molecules to treat COPD, there is a need for timely information from high-quality studies on both the effectiveness and safety of single drugs alone, and their combination. Efforts should be made to interpret and summarise findings originating from different research approaches, i.e. randomised controlled trials and observational studies, to guide decision-making from both clinical and drug-regulatory perspectives [28, 29]. COPD patients with concomitant CVD diseases represent a big everyday challenge for clinicians. Cardiologists and pulmonologists should work together in the context of an integrated care approach to optimise the management of the complex multimorbid COPD population [30].

\section{References}

1 Global Strategy for the Diagnosis, Management, and Prevention of COPD. Global Initiative for Chronic Obstructive Lung Disease (GOLD) 2016. Available from: http://goldcopd.org/global-strategy-diagnosis-managementprevention-copd-2016/

2 Farne HA, Cates CJ. Long-acting beta2-agonist in addition to tiotropium versus either tiotropium or long-acting beta2-agonist alone for chronic obstructive pulmonary disease. Cochrane Database Syst Rev 2015; 10: CD008989.

3 Lipworth B, Wedzicha J, Devereux G, et al. Beta-blockers in COPD: time for reappraisal. Eur Respir J 2016; 48: $880-888$

4 Singh S, Loke YK, Enright $\mathrm{P}$, et al. Pro-arrhythmic and pro-ischaemic effects of inhaled anticholinergic medications. Thorax 2013; 68: 114-116.

5 Lahousse L, Verhamme KM, Stricker BH, et al. Cardiac effects of current treatments of chronic obstructive pulmonary disease. Lancet Respir Med 2016; 4: 149-164.

6 Suissa S, Dell'Aniello S, Ernst P. Concurrent use of long-acting bronchodilators in COPD and the risk of adverse cardiovascular events. Eur Respir J 2017; 49: 1602245. 
7 Mullerova H, Agusti A, Erqou S, et al. Cardiovascular comorbidity in COPD: systematic literature review. Chest 2013; 144: 1163-1178.

8 Chen W, Thomas J, Sadatsafavi M, et al. Risk of cardiovascular comorbidity in patients with chronic obstructive pulmonary disease: a systematic review and meta-analysis. Lancet Respir Med 2015; 3: 631-639.

9 Barnes PJ. Senescence in COPD and its comorbidities. Annu Rev Physiol 2017; 20.1-20.23.

10 Mentz RJ, Kelly JP, von Lueder TG, et al. Noncardiac comorbidities in heart failure with reduced versus preserved ejection fraction. J Am Coll Cardiol 2014; 64: 2281-2293.

11 Rushton CA, Satchithananda DK, Jones PW, et al. Non-cardiovascular comorbidity, severity and prognosis in non-selected heart failure populations: a systematic review and meta-analysis. Int J Cardiol 2015; 196: 98-106.

12 Mohammed J, Meeus M, Derom E, et al. Evidence for autonomic function and its influencing factors in subjects with COPD: a systematic review. Respir Care 2015; 60: 1841-1851.

13 Roque AL, Valenti VE, Massetti T, et al. Chronic obstructive pulmonary disease and heart rate variability: a literature update. Int Arch Med 2014; 43: 1-8.

14 Corbo GM, Inchingolo R, Squeglia GA, et al. C-reactive protein, lung hyperinflation and heart rate variability in chronic obstructive pulmonary disease. A pilot study. COPD 2013; 200-207.

15 Lanza GA, Guido V, Galeazzi MM, et al. Prognostic role of heart rate variability in patients with a recent acute myocardial infarction. Am J Cardiol 1998; 82: 1323-1328.

16 Grassi G, Seravalle G, Mancia G. Sympathetic activation in cardiovascular disease: evidence, clinical impact and therapeutic implications. Eur J Clin Invest 2015; 45: 1367-1375.

17 Wu YK, Huang CY, Yang MC, et al. Effect of tiotropium on heart rate variability in stable chronic obstructive pulmonary disease patients. J Aerosol Med Pulm Drug Deliv 2015; 28: 100-105.

18 Haarmann $\mathrm{H}$, Mohrlang $\mathrm{C}$, Tschiesner $\mathrm{U}$, et al. Inhaled $\beta$-agonist does not modify sympathetic activity in patients with COPD. BMC Pulm Med 2015; 15: 46.

19 Ponikowski P, Voors AA, Anker SD, et al. 2016 ESC Guidelines for the diagnosis and treatment of acute and chronic heart failure. Eur Heart J 2016; 18: 891-975.

20 Mureddu GF, Agabiti N, Rizzello V, et al. Prevalence of preclinical and clinical heart failure in the elderly. A population-based study in Central Italy. Eur J Heart Fail 2012; 14: 718-729.

21 Miljkovik LV, Spiroska V. Heart failure with preserved ejection fraction - concept, pathophysiology, diagnosis and challenges for treatment. Open Access Maced J Med Sci 2015; 3: 521-527.

22 Yancy CW, Jessup M, Bozkurt B, et al. 2016 ACC/AHA/HFSA Focused Update on New Pharmacological Therapy for Heart Failure: An Update of the 2013 ACCF/AHA Guideline for the Management of Heart Failure: A Report of the American College of Cardiology/American Heart Association Task Force on Clinical Practice Guidelines and the Heart Failure Society of America. Circulation 2016; 134: e282-293.

23 Güder G, Brenner S, Störk S, et al. Chronic obstructive pulmonary disease in heart failure: accurate diagnosis and treatment. Eur J Heart Fail 2014; 16: 1273-1282.

24 McAllister DA, Maclay JD, Mills NL, et al. Diagnosis of myocardial infarction following hospitalisation for exacerbation of COPD. Eur Respir J 2012; 39: 1097-1103.

25 Hawkins NM, Virani S, Ceconi C. Heart failure and chronic obstructive pulmonary disease: the challenges facing physicians and health services. Eur Heart J 2013; 34: 2795-2803.

26 Suzuki T, Lyon A, Saggar R, et al. Biomarkers of acute cardiovascular and pulmonary diseases. Eur Heart J Acute Cardiovasc Care 2016; 5: 416-433.

27 Shah AS, Langrish JP, Nair H, et al. Global association of air pollution and heart failure: a systematic review and meta-analysis. Lancet 2013; 382: 1039-1048.

28 De Soyza A, Calverley PM. Large trials, new knowledge: the changing face of COPD management. Eur Respir J 2015; 45: 1692-1703.

29 Kardos P, Worsley S, Singh D, et al. Randomized controlled trials and real-world observational studies in evaluating cardiovascular safety of inhaled bronchodilator therapy in COPD. Int J Chron Obstruct Pulmon Dis 2016; 11: 2885-2895.

30 Roversi S, Fabbri LM, Sin DD, et al. Chronic obstructive pulmonary disease and cardiac diseases. An urgent need for integrated care. Am J Respir Crit Care Med 2016; 194: 1319-1336. 